\title{
Molecular psychiatry at the millennium
}

The beginning of the fifth volume of our journal and the start of the new millennium present a good occasion to reflect on the course of the journal and of our field.

Both the field and the journal have grown immensely during the last five years. The journal has now an impact factor of 4.8, which is the fourth in the Institute of Scientific Information's (ISI) listing for psychiatry. ISI has developed the 'immediacy index,' which indicates how many times the articles published by a journal are cited in the same year that they are published. The ISI calls this a measure of how 'hot' a journal is, because it indicates how rapidly others cite material that a journal publishes. Our very high immediacy index surpasses those of well-known new and established journals; we are proud to be the only journal to have one of the top five immediacy indices both in psychiatry and in neurosciences. This indicates that we are truly fostering the growth of a neurosciencebased psychiatry that is widely cited-some of our articles have already been cited over 70 times.

As we grow, we continue to offer rapid peer review and publication and a lively mixture of basic and clinical sciences that is of interest to researchers and clinicians in psychiatry and related areas. As an indication of our increasing popularity, we are delighted that the International Society of Psychiatric Genetics chose Molecular Psychiatry to publish the abstracts of its recent meeting in Monterey, California.

Molecular Psychiatry will continue to publish our highly successful mixture of shorter and longer articles, as well as various types of sections. A new section is our Millennium Articles. These are state of the art reviews and commentaries on topics of particular relevance. Some of these articles include both a retrospective look, while others are more forward looking. We have carefully commissioned a mixture of both short and longer pieces with various foci that catch the light of current thought in our field from different angles. The comments that I have received from readers, authors, and members of our editorial board are that a key feature of the journal is its openness to new, high-quality ideas. This feature is embodied in the Millennium articles. A common thread of the articles is their relevance both to the past and to the future of our field. We were indeed fortunate to have had someone with the experience and wisdom of George Heninger to write the first paper in the series on the opport-

Correspondence: Dr J Licinio, UCLA Department of Psychiatry \& Biobehavioral Sciences, 3357A Gonda (Goldschmied) Center, 695 Charles E Young Dr South, Box 951761, Los Angeles, CA 900951761, USA. E-mail: licinio@ucla.edu unities and limitations of psychiatric research in the 21st century.

What about the field itself? Where do we stand? Where are we going? It is noteworthy that as the century ends, we are for the first time seeing solid scientific data supporting earlier, turn of the (last) century ideas on the importance of environmental factors in psychiatric disorders. Specifically, in our first volume we published an article by Bode et al from the Robert Koch-Institut and the Free University of Berlin, ${ }^{1}$ reporting the discovery of the human Borna virus, a possible cause of depression. In our March 1999 issue we published an original research article by Agid et al from the Hadassah-Hebrew University Medical Center (Jerusalem), ${ }^{2}$ showing in a case control study that loss of a parent during childhood significantly increased the likelihood of developing major depression during adult life. It is important to note that contemporary research utilizing rigorous methodology supports not only the genetic, but also the environmental contributions to psychiatric disorders. The impact of these specific environmental factors, namely microorganisms and loss, on the course of psychiatric disorders was noted both by Julius Wagner-Jauregg (Nobel Prize for Physiology or Medicine, 1927) and by Sigmund Freud about a hundred years ago (for further commentaries on these points, see Licinio ${ }^{3,4}$ ).

In our work we become very involved in specific aspects of various psychiatric disorders. I sometimes find it helpful to stop and ask myself basic questions such as 'What is depression?', 'What is schizophrenia?' Are these a constellation of symptoms, of biological events, an umbrella for several diseases with similar presentations, or are they truly well-defined syndromes? Are psychiatric presentations the end result of specific and necessary combinations of genetic, developmental, and environmental events, or can they be the result of one factor-one loss, one trauma, one mutated gene? Having seen several hundred articles, submitted to our journal as well as published elsewhere, I have over the course of the last four years noticed a change in my feelings about the field. When the journal was launched in 1996, there was a general expectancy that very soon one mutation would be published in a high profile journal that would explain, at least in some individuals, diagnoses such as schizophrenia or major depression. That would be another confirmation of the 'one gene, one protein, one disease' perspective. However, the weeks became months, the months became years, and such a gene was not reported.

Meanwhile, hundreds of publications came out on the small, but significant association between psychi- 
atric disorders and alleles of many genes. Based on these papers one would assume that a variety of genes contribute-each in a minor, but significant mannerto psychiatric phenotypes. It is highly possible that dozens of susceptibility genes for diagnosis such as depression, schizophrenia, or obsessive-compulsive disorder, exist, none of which is necessary or sufficient to cause a disorder. Moreover, as will be discussed in one of our upcoming Millennium Articles, it has been hypothesized that one gene may contribute to more than one psychiatric disorder. Allellic variations in subsets of many genes, reaching some type of threshold, possibly in the context of psychosocial, infectious, or inflammatory stressors, may result in the fullblown clinical presentations that we see in our offices, hospitals, and sadly in our streets and in the penal system. Such complex models, where multitudes of effects caused by many genes, loss, trauma, viruses, and the body's own responses, may one day be integrated. What we are now doing in our field is to generate, association by association, finding by finding, article by article, the pieces of a complex puzzle that one day will be put together by well-prepared minds aided by powerful computational tools. Such models will parcel out the relative contributions of factors such as heredity, microbes, and loss, which for over a hundred years have been considered as mechanisms underlying psychiatric disorders. It is impossible to put any puzzle together unless one has all its pieces. I see the articles being published now, in Molecular Psychiatry and elsewhere, as such pieces of the puzzles that are psychiatric disorders. Some pieces are bigger and more exciting than others-however, all of them are required to fully resolve the puzzles.

Nevertheless, I still look avidly at each incoming paper, as well as at the table of contents of all good journals as soon as I get them, rapidly scanning article titles for the report that-finally-shows one genetic mutation that causes depression or schizophrenia.

It remains to be determined whether psychiatric disorders will be explained by one nucleotide substitution or by a highly complex, computerized model of scores of 'guilty' alleles, in the context of specific psychosocial, developmental, nutritional, infectious, and immune substrates. It is not only our curiosity that will be quenched when either possibility is fulfilled. The lives of patients with psychiatric disorders, so many of them severely impaired for decades, will be the primary beneficiaries of progress.

J Licinio

University of California, Los Angeles School of Medicine

Department of Psychiatry and Biobehavioral Sciences Los Angeles, CA 90095-1761, USA

\section{References}

1 Bode L, Durrwald R, Rantam FA, Ferszt R, Ludwig H. First isolates of infectious human Borna disease virus from patients with mood disorders. Mol Psychiatry 1996; 1: 200-212.

2 Agid O, Shapira B, Zislin J, Ritsner M, Hanin B, Murad H et al. Environment and vulnerability to major psychiatric illness: a case control study of early parental loss in major depression, bipolar disorder and schizophrenia. Mol Psychiatry 1999; 4: 163-172.

3 Licinio J. What makes one tic. Science 1999; 286: 56-57.

4 Licinio J. Expressing Freudian influences. Science 1999; 282 2197-2198. 